\title{
A Survey of Data Recording Procedures at New York City Emergency Departments
}

\author{
Jessica Sell ${ }^{\star 1}$ and Alyssa Wong ${ }^{2}$ \\ ${ }^{1}$ NYC Department of Health and Mental Hygiene, Long Island City, NY, USA; ${ }^{2}$ Columbia University, Mailman School of Public Health, \\ New York, NY, USA
}

\section{Objective}

To describe the development, implementation, and analysis of a hospital based emergency department (ED) survey and site visit project conducted by the New York City (NYC) Department of Health and Mental Hygiene (DOHMH).

\section{Introduction}

Data is collected daily by the DOHMH from 49 of the 52 NYC EDs, representing approximately $95 \%$ of all ED visits in NYC. Variability in data fields between and within EDs has been noticed for some time. Differences in chief complaint (CC) characteristics and inconsistent availability of data elements, such as disposition and diagnosis, suggest that procedures, coding practices and health information systems (HIS) are not standardized across all NYC EDs, and may change within EDs. These differences may have an unapparent effect on the DOHMH's ability to consistently categorize ED visits into syndrome groupings, which may alter how syndromic trends are analyzed. Prior to this project, the DOHMH had no method in place to regularly capture, evaluate or utilize this level of ED-specific information.

\section{Methods}

A member of the DOHMH contacted all 49 EDs to request a brief interview with the ED director, administrator and/or appropriate staff. A questionnaire was designed to collect the following information about each ED: the clinical and administrative HIS used to collect patient information and report it to the DOHMH (including any recent system changes); $\mathrm{CC}$ coding practices (i.e. who records the $\mathrm{CC}$, and into which HIS, and in what format); disposition and diagnosis recording practices and availability. Questions regarding hospital specific trends and characteristics were also included. Interviews were conducted in person by two members of the DOHMH.

Information from the survey was compiled into a Microsoft Excel spreadsheet by the interviewers. A descriptive analysis was performed comparing and detailing HIS used, CC coding practices, and recording procedures for disposition and diagnosis. A member of the DOHMH followed up with ED staff and IT personnel to resolve any outstanding data quality issues.

\section{Results}

All 49 EDs were contacted and interviewed. A median of 43 days (ranging from 7 to 167) elapsed between the initial attempt to contact the ED director, and the completion of the interview. All interviews lasted approximately 40 minutes.

According to the results of the survey, the DOHMH receives information from the clinical HIS from approximately $20 \%$ of EDs, from the administrative HIS from approximately $70 \%$ of the EDs, and approximately $10 \%$ of the EDs did not know which system was used to generate the daily reports sent to the DOHMH. Nearly $100 \%$ of the EDs reported that the chief complaint was entered into the clinical HIS by a triage nurse. However, it is not known who records the $\mathrm{CC}$ into the administrative system. Four EDs reported that a dropdown menu is used to record CC into the clinical HIS, 23 EDs CC is in free-text format, and $22 \mathrm{EDs}$ CC is a combination of free-text and drop-down format.

Diagnosis was recorded by the physician at $45 \%$ of the EDs, and by other staff, including nurses and clerks, at $55 \%$ of the EDs. Two thirds of the EDs reported a lag time of less than one week between the visit and assignment of diagnosis codes. Disposition is recorded by the physician at $80 \%$ of EDs. Discharge disposition is often required for a patients chart to be considered complete.

As a result of the visits the DOHMH was able to better understand problems that cause routine data quality problems (e.g. missing data or unusable data) by hospital and identify methods to improve those problems. Missing and up to date disposition codebooks were obtained from hospitals. Current hospital contacts were identified for follow up.

Discussions with hospital personnel regarding specific trends, characteristics and interests helped to strengthen the relationship the DOHMH has with the hospital ED staff.

\section{Conclusions}

Differences in practices, procedures, and HIS used can lead to variability in data quality and characteristics which may affect the ability to categorize visits into effective syndrome groupings and understand trends. Further research is needed to develop an improved method for analyzing ED data that takes ED-specific characteristics into consideration. Additionally, it is important to establish good working relationships with key members of each ED's staff in the event of a possible outbreak, and in keeping up to date on any changes within each ED that may affect data quality.

Keywords

Emergency Department; Syndromic Surveillance; Coding Practices

\section{Acknowledgments}

NYC DOHMH

Alfred P. Sloan Foundation

*Jessica Sell

E-mail: jsell@health.nyc.gov 